EPJ Web of Conferences 64, 08007 (2014)

DOI: $10.1051 /$ epjconf/ 20146408007

(C) Owned by the authors, published by EDP Sciences, 2014

\title{
Cool, warm and hot outflows from CTTS: The FUV view of DG Tau
}

\author{
P. C. Schneider ${ }^{1, a}$, J. Eislöffel ${ }^{2}$, M. Güdel ${ }^{3}$, H.M. Günther ${ }^{4}$, G. Herczeg ${ }^{5}$, J. Robrade ${ }^{1}$, and \\ J.H.M.M. Schmitt ${ }^{1}$
}

${ }^{1}$ Hamburger Sternwarte, Gojenbergsweg 112, 21029 Hamburg, Germany

${ }^{2}$ Thüringer Landessternwarte Tautenburg, Sternwarte 5, 07778 Tautenburg, Germany

${ }^{3}$ Department of Astrophysics, Türkenschanzstrasse 17, A-1180 Vienna, Austria

${ }^{4}$ Harvard-Smithsonian Center for Astrophysics, 60 Garden Street, Cambridge, MA, USA

${ }^{5}$ Kavli Institute for Astronomy and Astrophysics, Peking University, Beijing, China

\begin{abstract}
Classical T Tauri stars (CTTSs) drive strong outflows with temperatures from about $10^{3} \mathrm{~K}$ up to a few $10^{6} \mathrm{~K}$. These outflows regulate the angular momentum balance and are therefore tightly related to the accretion process. However, the outflow driving and heating mechanisms are not well understood. We present new HST data of the "prototypical" jet-driving CTTS DG Tau tracing the low-temperature outflow with fluorescently excited far-UV molecular hydrogen emission and the high-temperature part with $\mathrm{C}$ iv emission. We find that the spatial distribution of the low-temperature plasma is V-shaped consistent with molecular disk-wind models. Low-velocity shocks $\left(v_{\text {shock }} \sim 30 \mathrm{~km} \mathrm{~s}^{-1}\right)$ are probably the pumping source for the FUV $\mathrm{H}_{2}$ lines. The hot plasma $\left(T>10^{5} \mathrm{~K}\right)$ is located close to the jet axis at a distance of $40 \mathrm{AU}$ from the driving source and spatially offset from standard (optical) jet-tracers like [S II] or [O I]. It does not show any hints for proper-motion contrasting typical jet properties. The high-temperature plasma is unlikely caused by a hot stellar wind and we propose that the stationary heating is caused by internal shocks or magnetic reconnection.
\end{abstract}

\section{Introduction}

In young pre-stellar systems, accretion is accompanied by outflow activity (e.g., [1]). To maintain accretion from a circumstellar disk, angular momentum needs to be redistributed within the system. Rotating outflows can accomplish this task [2]. The fact that the mass-loss though the outflow is about $10 \%$ of the accretion rate is regarded as the observational manifestation of the tight physical connection between accretion and outflows [3]. However, the initial structure of these outflows is not well understood so that the connection between accretion and outflows remains somewhat vague. In particular the launching and collimation of the outflow still presents some severe puzzles. Therefore, observations of the outflow structure as close as possible to the driving source are needed. In the past decade, X-ray observations showed that the outflows reach much higher temperatures than

\footnotetext{
ae-mail: christian.schneider@hs.uni-hamburg.de
} 


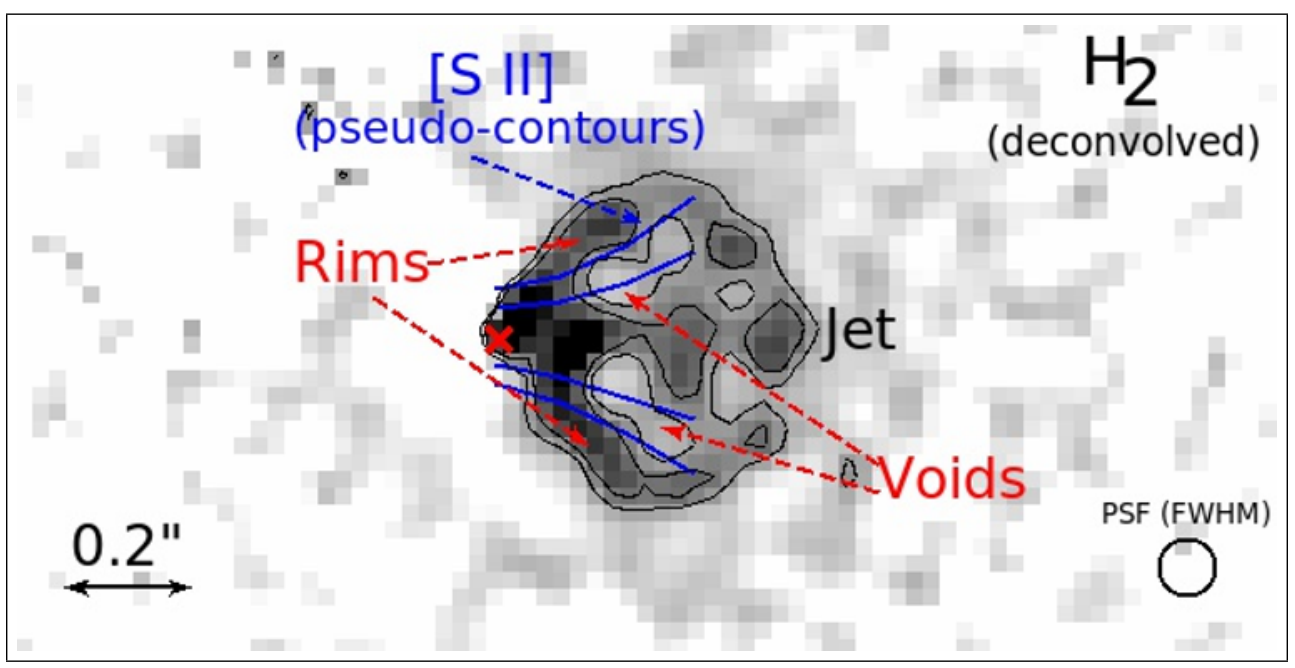

Figure 1. FUV $\mathrm{H}_{2}$ emission from the DG Tau system. The forward jet direction is towards the right $\left(\mathrm{PA} \sim 225^{\circ}\right)$. The [S II] $\lambda 6731$ pseudo-contours were obtained from the 1999 HST data presented by $[11,12]$. They enclose $68 \%$ and $90 \%$ of the emission perpendicular to the jet axis. The $\mathrm{H}_{2}$ emission has been deconvolved using the Lucy-Richardson method. See [10] for details.

previously anticipated (e.g., [4-7]). Furthermore, multi-epoch studies showed that the X-ray emitting plasma $\left(T \gtrsim 3 \times 10^{6} \mathrm{~K}\right)$ is stationary in some systems which is also not predicted by standard theories. Here, we present Hubble Space Telescope (HST) data of the outflow from the single classical T Tauri star (CTTS) DG Tau. Specifically, we focus on the temperature range from $10^{3}$ to $10^{5} \mathrm{~K}$ to gain further insight into the connection of the stationary X-ray emission with the well studied, lower temperature part.

\section{Cool: Molecular Hydrogen emission}

FUV molecular hydrogen line emission is fluorescently excited by Ly $\alpha$ photons ${ }^{1}$. It traces the amount of warm $(\sim 2000 \mathrm{~K})$ molecular hydrogen and local Ly $\alpha$ radiation field. Figure 2 shows the UV $\mathrm{H}_{2}$ emission associated with outflow of DG Tau. Two bright rims enclosing an angle of about $90^{\circ}$ and emission close to the jet axis are clearly visible in the image. Bright $H_{2}$ rims are also visible in near-IR images of the DG Tau jet [8]. We interpret the spatial morphology of the FUV $\mathrm{H}_{2}$ emission as evidence for a warm disk wind heated predominately by ambipolar-diffusion (see models by [9]). The position of the $\mathrm{H}_{2}$ emission, i.e., located at greater distances from the jet axis than the faster [S II] $\lambda 6731$ also points towards a disk wind origin (see [10]).

\section{Warm: Optical line emission}

The warm $\left(T \sim 10^{4} \mathrm{~K}\right)$ part of the jet is traced by forbidden emission lines (FELs). Our optical HST long-slit spectrum shows two knots within the innermost $140 \mathrm{AU}$ (see Fig. 2). The faster knot at 0.3 $\operatorname{arcsec}(63 \mathrm{AU})$ is located farther from the driving source than the inner, stationary X-ray emission

\footnotetext{
${ }^{1}$ Some minor fraction of the $H_{2}$ emission might come from fluorescence routes excited by $\mathrm{C}$ IV photons.
} 

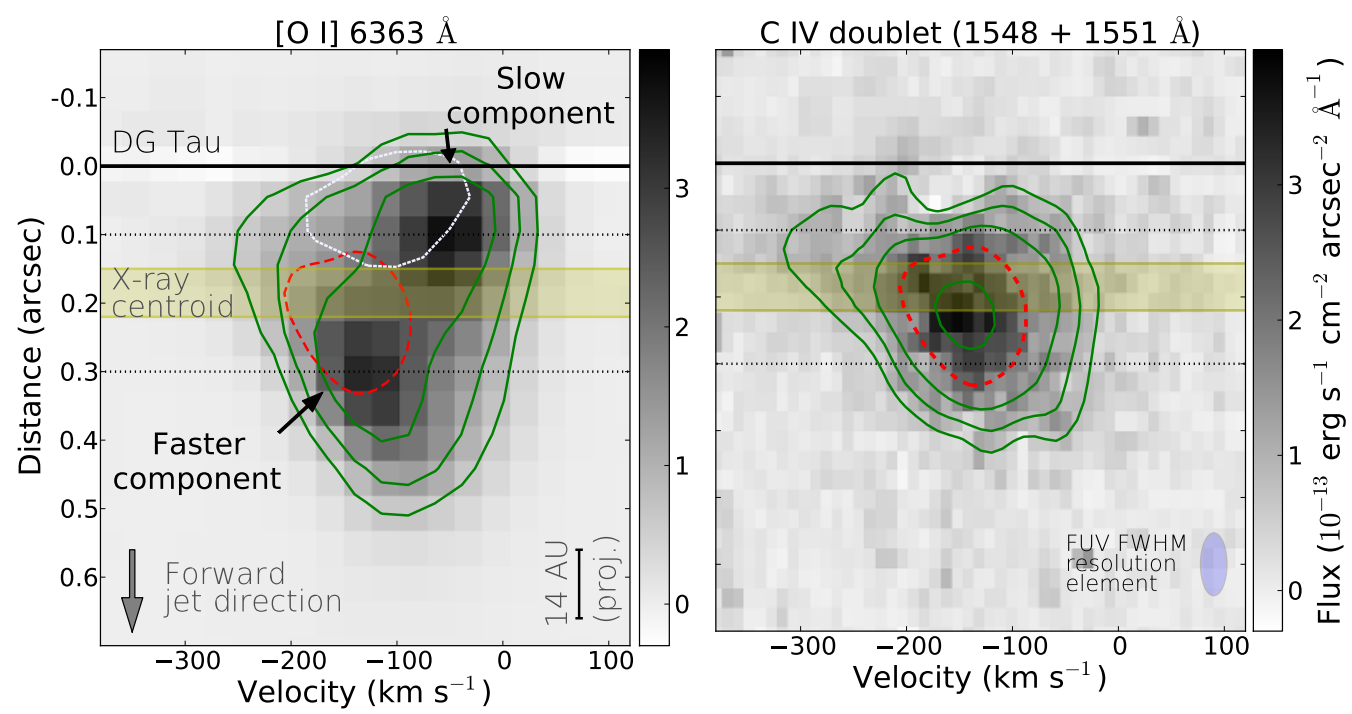

Figure 2. Long-slit spectra of the DG Tau outflow. The slit-width is $0.2 \operatorname{arcsec}$ and the slit was aligned along the jet axis. The position of the X-ray emitting plasma is indicated. The dotted lines indicate the distance of the two optical knots in both panels. Left: Warm material at $T \sim 10^{4} \mathrm{~K}$ traced by [O I] $\lambda 6363$ emission. The stellar continuum emission has been removed. Right: The hot part $\left(T \sim 10^{5} \mathrm{~K}\right)$ of the outflow as traced by FUV C IV $\lambda \lambda 1548,1551$ emission. Contamination by $H_{2}$ emission has been removed.

[6, 13]. The slower one at 0.1 " (21 AU) is located closer to DG Tau than the X-ray emission. Its velocity is too low to be directly related with the X-ray emission. At the centroid position of the X-ray emission no (or very little) low temperature $\left(T \sim 10^{4} \mathrm{~K}\right)$ plasma is present. Comparing our new data with the data of $[11,12]$ we find that the inner, low-velocity emission component appears stationary as previously suggested from ground-based data [14]. However, its velocity is too high for a photo-evaporating wind. Its stationary nature indicates that this inner, low-velocity component is not associated with individual knots observed farther out in the DG Tau system. Thus, no plasma component with a temperature around $10^{4} \mathrm{~K}$ is directly related to the X-ray emitting plasma.

\section{Hot: FUV emission}

We trace the hot $\left(T \sim 10^{5} \mathrm{~K}\right)$ jet with $\mathrm{C}$ IV emission which dominates the radiative cooling at those temperatures. Figure 2 shows the $\mathrm{C}$ IV position-velocity diagram. It demonstrates that the spatial distribution of the hot plasma overlaps with the centroid of the X-ray emission $\left(T>10^{6} \mathrm{~K}\right)$, but is offset from the lower temperature optical emission. This indicates that the $\mathrm{C}$ IV emission is likely associated with the X-ray emitting plasma and traces a different jet component than the lower temperature outflow. Comparison with archival data suggests that the $\mathrm{C}$ IV emission is stationary as well and not directly associated with moving knots (see [15] for details). The stationary appearance might originate from an initially hot outflow, e.g., a stellar wind or from a stationary heating mechanism. A stellar wind, however, cools too rapidly to explain the offset observed for the $\mathrm{C}$ IV emission. Therefore, we favor an internal heating mechanism at some distance to the driving source. Possibilities are shocks associated with the collimation process or magnetic heating through reconnection events. 
Furthermore, no emission is seen at the stellar position which demonstrates that a correlation between $\mathrm{C}$ IV luminosity and accretion rate might be indirect via the accretion-outflow relation in some objects.

\section{Conclusions}

CTTS drive different kinds of outflows. The low-temperature part $\left(10^{3}-10^{4} \mathrm{~K}\right)$ is likely caused by the disk wind although probably different mechanisms are responsible for the excitation, i.e., the lowest temperature part $\left(10^{3} \mathrm{~K}\right)$ is heated by ambipolar-diffusion while the warm part $\left(10^{4} \mathrm{~K}\right)$ is heated by internal shocks and possesses clear proper-motion. This interpretation is consistent with the spatial morphology of the $\mathrm{H} 2$ emission and can explain the optical/near-IR emission. The emission from the high-temperature plasma is spatially offset from the low-temperature part; C IV and X-ray emission are co-spatial and likely stationary in position. This indicates that a different mechanism is responsible for their heating and that they are not associated with the stochastic shocks which are seen as emission from lower temperature plasma. The $\mathrm{C}$ IV data rule out an initially hot outflow, e.g., a stellar wind, as the origin for the hot outflow. Possibilities for its origin are standing shocks and magnetic reconnection events.

\section{References}

[1] J. Bally, B. Reipurth, C.J. Davis, Protostars and Planets V pp. 215-230 (2007)

[2] R.E. Pudritz, R. Ouyed, C. Fendt, A. Brandenburg, Protostars and Planets V pp. 277-294 (2007), arXiv:astro-ph/0603592

[3] P. Hartigan, S. Edwards, L. Ghandour, ApJ 452, 736 (1995)

[4] F. Favata, C.V.M. Fridlund, G. Micela, S. Sciortino, A.A. Kaas, A\&A 386, 204 (2002), arXiv: astro-ph/0110112

[5] M. Güdel, S.L. Skinner, K.R. Briggs, M. Audard, K. Arzner, A. Telleschi, ApJL 626, L53 (2005), arXiv: astro-ph/0505178

[6] P.C. Schneider, J.H.M.M. Schmitt, A\&A 488, L13 (2008), 0807 . 2156

[7] P.C. Schneider, H.M. Günther, J.H.M.M. Schmitt, A\&A 530, A123 (2011), 1105.1663

[8] T.L. Beck, P.J. McGregor, M. Takami, T.S. Pyo, ApJ 676, 472 (2008), 0711 . 3844

[9] D. Panoglou, S. Cabrit, G. Pineau Des Forêts, P.J.V. Garcia, J. Ferreira, F. Casse, A\&A 538, A2 (2012), 1112.3248

[10] P.C. Schneider, J. Eisloeffel, M. Guedel, H.M. Guenther, G. Herczeg, J. Robrade, J.H.M.M. Schmitt, ArXiv e-prints (2013), 1307.2846

[11] F. Bacciotti, R. Mundt, T.P. Ray, J. Eislöffel, J. Solf, M. Camezind, ApJL 537, L49 (2000), arXiv: astro-ph/0005463

[12] F. Bacciotti, T.P. Ray, R. Mundt, J. Eislöffel, J. Solf, ApJ 576, 222 (2002), arXiv: astro-ph/0206175

[13] M. Güdel, M. Audard, F. Bacciotti, J.S. Bary, K.R. Briggs, S. Cabrit, A. Carmona, C. Codella, C. Dougados, J. Eislöffel et al., The Bipolar X-Ray Jet of the Classical T Tauri Star DG Tau, in 16th Cambridge Workshop on Cool Stars, Stellar Systems, and the Sun, edited by C. Johns-Krull, M.K. Browning, A.A. West (2011), Vol. 448 of Astronomical Society of the Pacific Conference Series, p. 617, 1101.2780

[14] C. Lavalley, S. Cabrit, C. Dougados, P. Ferruit, R. Bacon, A\&A 327, 671 (1997)

[15] P.C. Schneider, J. Eislöffel, M. Güdel, H.M. Günther, G. Herczeg, J. Robrade, J.H.M.M. Schmitt, A\&A 550, L1 (2013), 1212.6363 